\title{
Comparison of reasorbeable mini anchor versus non reasorbeable: histological evaluation in an experimental rabbit model
}

\author{
Alex Pontini ${ }^{1}$ \\ Ivan Munivrana ${ }^{1}$ \\ Maria Luisa Valente ${ }^{2}$ \\ Franco Bassetto ${ }^{1}$ \\ 1 Department of Medico-Surgical Specialties, Hand \\ Unit, Institute of Plastic Surgery, University of \\ Padova, Italy \\ 2 Department of Diagnostic Medical Sciences, Sec- \\ tion of Special Pathology, University of Padova, \\ Italy
}

Corresponding author:

Alex Pontini

Department of Medico-Surgical Specialties, Hand Unit

Institute of Plastic Surgery, University of Padova

Via Giustiniani, 2

35100 Padova, Italy

E-mail: alex.pontini@libero.it

\section{Summary}

Purpose. Experimental animal study to evaluate the osseo integration, inflammatory response, dislocation and the reabsorption timing of the reasorbeable Mitek ${ }^{\circledR}$ mini anchor, in comparison with the non reasorbeable titanium based.

Methods. Twenty rabbits were used: divided into two groups of ten. Each animal underwent sectioning and reinsertion of the Achilles tendon bilaterally, using a reasorbeable mini anchor on one side and a controlateral non reasorbeable mini anchor. The first group was sacrificed after $\mathbf{4 0}$ days and the other after 120 days. The bone tendon complex was subjected to histological study. Results. No histological and statistical significative difference were observed in each group, with a lower inflammation response in the reasorbeable implant. Electronic microscopy evaluation demonstrates good stability of the implant in each group. At day 120 the reasorbeable anchors were not yet disappeared.

Conclusion. Reasorbeable Mitek mini anchors are to be considered to guarantee a similar response and similar bone stability than non reasorbeable ones.

KEY WORDS: anchor, reabsorption, tendon, osseointegration, rabbit.

\section{Introduction}

The use of suture anchors has been copiously reported in the literature for the attachment of soft tissues to cortical and spongy bone ${ }^{1}$. Orthopedic use of such suture include reconstruction of rotator cuff, Bankart's lesion's repair, meniscus tears or collateral ligament injuries treatment and different type of extremity injuries ${ }^{2-4}$. In the last few years their application has been expanded also to head and neck reconstruction and hand surgery, including various tendon injuries ${ }^{5,6}$. Actually suture anchors are produced in a lot of different shapes, design and sizes, even reasorbeable (polylactic) than non reasorbeable (titanium) anchors. Different advantages of bioabsorbable implants in comparison with traditional metallic implants have been described ${ }^{7,8}$. Reasorbeable implants seems to reduce stress shielding because their properties to gradually transfer load as they degrade. Depending of any specific application the bioabsorbable polymers can be also engineered to provide the desiderable degradation profile.

Generally reasorbeable implant allows to reduce a second procedure for its removal and could facilitate a needed revision surgery. Use of foreign implants require also control done by magnetic resonance imaging (MRI) and bio asorbeable implants offer a very low distortion rate.

First studies on bioabsorbable materials was published in 1966, when Kulkarni et al. published a report on the histological response and the degradation of poly-L-lactic acid (PLLA) powder implanted in guinea pigs and rats $^{9}$. It was found that the polymer was nontoxic, nontissue reactive, and degraded slowly. The reasorbeable material of Mytek mini anchor is based on Poly-L-lactic acid (PLLA), that is the $L$ isomer of polylactic acid. It is hydrophobic and crystalline and has an extended degradation time.

Traditional mitek mini anchors are composed of a titanium alloy shaft, represented by titanium (90\%), aluminum $(6 \%)$ and vanadium $(4 \%)$. Their measure, even reasorbeable that not reasorbeable are $1.8 \mathrm{~mm}$ in diameter and $5.0 \mathrm{~mm}$ long.

Suture anchors design were thought to allow their placement into a bone hole obtained by a mechanical predrilling. Then the fixation of the anchor within the bone is provided by engaging their specific structures (arcs, barbs, or threads) attached to the anchor within the bone. A suture, generally reasorbeable, is available to be attached to the soft tissue, so they are directly fixed to the anchor. Suture anchors, have been 
shown to be stronger than the attached sutures $^{10}$ Fixation is more secure than periosteal suture because the soft tissue could be strongly attached to a bony embedded anchor. In hand surgery, the development in the last few years of the Mitek mini and micro suture anchor (Mitek Inc, Norwood, MA) to repair tendons, ligaments and the nearest structures, has permitted an important alternative to traditional pullout technique, both transosseus ${ }^{11}$ and subcutaneous, according to Mantero technique ${ }^{12}$.

However, the disadvantage of a permanent implant (titanium) could conduce to a secondary surgery for its removal in case of dislocation, fracture, infections or inflammatory response to a foreign body ${ }^{13}$. The reasorbeable implant in polylactic acid seems to avoid this complication ${ }^{14}$ but its utilisation in hand surgery is actually impeded and debated because of the poor scientific literature. The aim of this experimental study is to histological evaluate osseointegration properties of the two different types of Mitek mini anchors at different phases of healing.

\section{Materials and methods}

This study was approved by the Research Ethics Committees of the National Ministry of Health, and it was conduced in accordance with the principles of the Declaration of Helsinki regarding experimentation involving animals, in our Experimental Surgery Centre. The sample consisted of 20 male rabbits (Oryctolagus cuniculus) of the New Zealand White breed, weighing between 2200 and $3300 \mathrm{~g}$. The animals were kept in the vivarium of our Experimental Surgery Centre, housed in adequately metal cages, with necessary hydratation and food and weekly controlled from Veterinary Doctors of our University. The animals were divided randomly into two groups composed of 10 animals each: group A ( 40 days of survival) and group B (120 days of survival). Anaesthesia was administered with an intra muscle injection of $0,4 \mathrm{ml} / \mathrm{kg}$ of tiletamine chloridhrate and zolazepam in

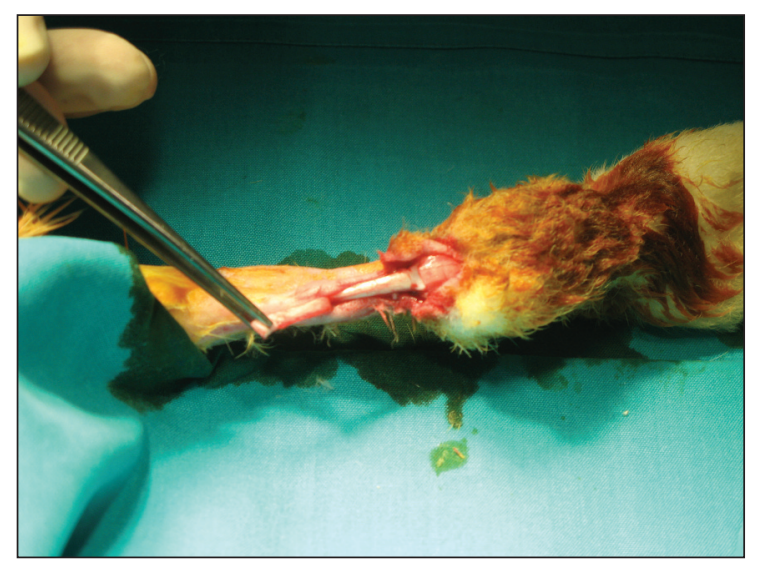

Figure 1. In each animal an incision exposed the Achilles tendon and it was sectioned adjacent to insertion into the bone. the proximal region of pelvic limbs, joint to a local injection of lidocaine, applied to the incision site.

Antibiotic prophylaxis was administered, consisting of ofloxacin at dose of $5 \mathrm{mg} / \mathrm{kg}$ intramuscularly at the time of inducing anesthesia, and this was maintained for 6 days. Analgesia during the postoperative period, was obtained by $3 \mathrm{mg} / \mathrm{kg}$ of ketorolac, maintaining for 48-72 hours. After the onset of anesthesia, all the animals were subjected to surgical procedure. The area was accurately shaved with exposure of the skin in the calcaneus region and disinfected with povidone-iodine solution. An "S" incision was performed to expose the Achilles tendon and then the tendons of both pelvic limbs were sectioned adjacent to their insertions into the bone ${ }^{15}$, reproducing a tendon injury with the loss of bone insertion (Fig. 1). The sectioned tendons were then reinserted by using two different types of Mitek mini anchor, one side randomly decided with a non reasorbeable one, and the other side with a reasorbeable one. Using a $1.3 \mathrm{~mm}$ diameter drill, 2 holes were made into the calcaneus bone: the anchor was then fixed into it and with the non reasorbeable suture joint to the anchor was performed a tendon repair according to Kessler technique with a good fixation of the Achilles tendon to the calcaneus bone (Fig. 2). Both techniques were performed during the same operation on each rabbit, one on each pelvic limb. Thus, the same number of reinsertion was obtained for each mini anchor on the right and on the left side. The skin was then closed using nylon 5/0 thread and the operative wound was covered with a sterilized gauze compress. These 20 animals were divided into 2 groups with different postoperative periods and sacrificed afterwards: group A, 40 days; group B, 120 days. Following drug-induced sacrifice, the respective Achilles tendons were identified and isolated ${ }^{16}$.

The specimens of the pelvic limb, composed of a part of calcaneus bone containing the anchor and the tendon fixed to the bone were then extracted (Fig. 3). These were draped in dampened gauze with a saline solution of $0,9 \%$ sodium chloride and then the gauze was removed and the specimens were immediately placed in $10 \%$ neutral-buffered formalin. The speci-

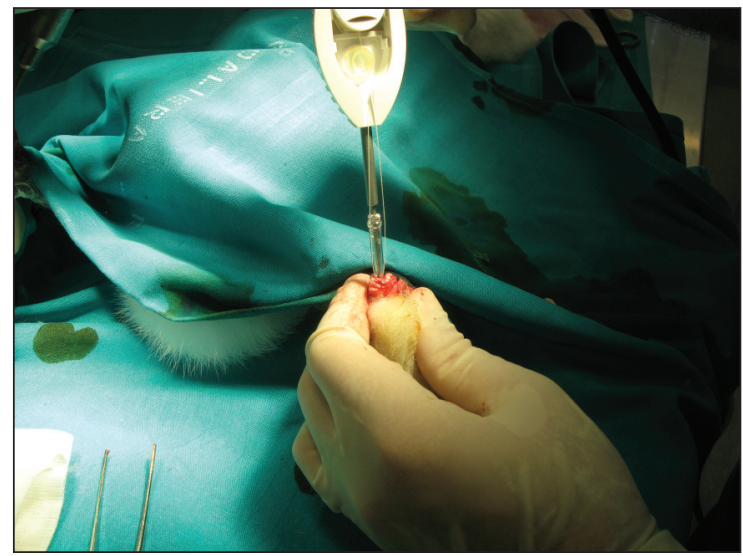

Figure 2. The anchor of each type was fixed in calcaneus bone and a tendon repair was performed. 


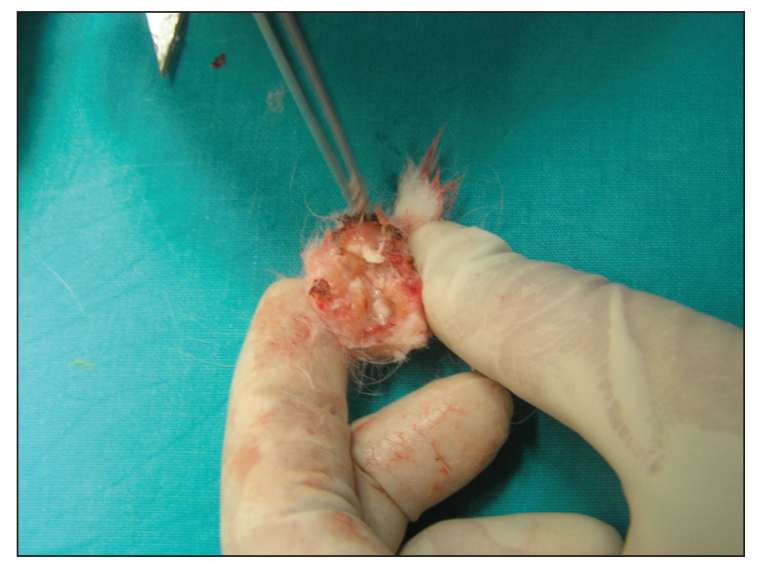

Figure 3. The specimens, composed of a part of bone containing the anchor and the tendon fixed to the bone were then extracted.

mens were subsequently sent to our Institute of Pathology. The undecalcified calcaneous bones were dehydrated via ascending alcohol concentrations, and then they were impregnated with a 1:1 mixture of alcohol/Technovit 7200 VLC, followed by infiltration with pure Technovit 7200 VLC (Heraeus Kulzer, Germany). According to the Säge-Schliff (sawing and grinding) technique ${ }^{17}$ the embedded specimens were sawed along the longinal axis of the mini-Anchors with an Exakt@ sawing machine to a thickness of about 100 micrometers and then ground with an Exact@grinding machine to a thickness of 10 micrometers. The polished sections were stained with Haematoxylin-Eosin (H\&E) and Masson-Goldner staining. Histological evaluation was performed on a Leica DM $4000 B$ microscope, equipped with a Leica DFC 320 electronic camera. Each specimen was evaluated for intervening fibrous connective tissue, presence or absence of Osseo integration, anchor particulation and fracture, inflammatory cell infiltration, in comparison with non reasorbeable titanium anchors and reasorbeable polylactic anchors.

\section{Results}

At the time of extraction, each suture from the anchor to the tendon, in both groups was clinically intact, and each anchor was firmly embedded in the bone, except in one case (group B) in which both anchors were dislocated, probably because of the movement of the animal that was referred during the whole post-operative period. Light microscopic evaluation revealed in Group A -with no significant difference between reasorbeable and non reasorbeable anchors, -that the anchor was circumscribed with the bone, the eyelet end of each suture anchor was always deep to the cortical surface of the bone, no inflammatory cell infiltrate or foreign body giant cell reactions were evident. No particulation or fracture was associated with any of the anchors (Figs. 4, 5). In group $B$, the microscopic evaluation demonstrates

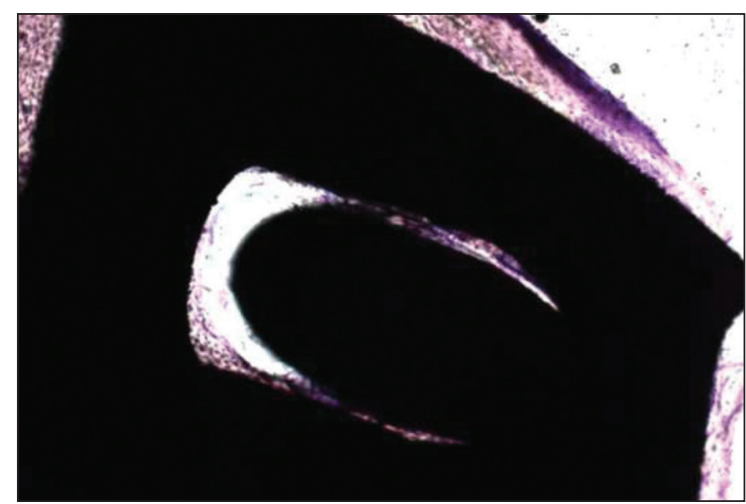

Figure 4. Group A. Titanium mini anchor implant at day 40. No difference with reasorbeable implant were observed.

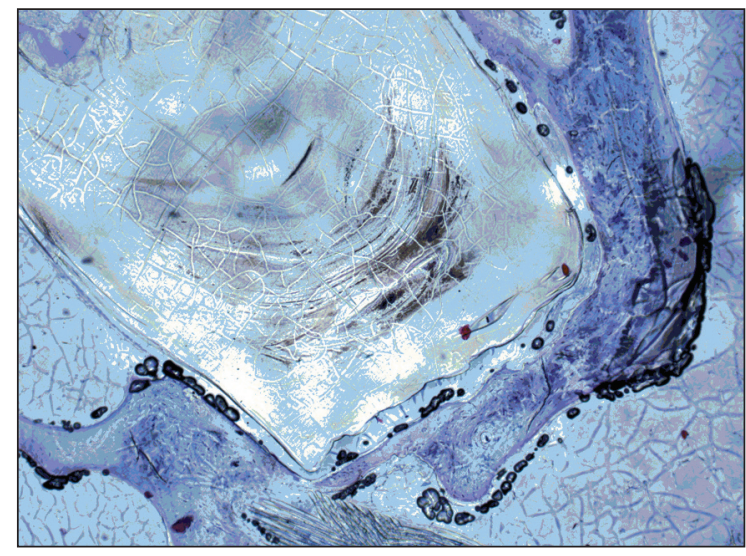

Figure 5. Group A. Polilactic mini anchor implant at day 40.

that titanium implants were good embedded in bone with no dislocation (Fig. 6). The reasorbeable anchors had not disappeared, including extensive regions of direct mineral apposition (Fig. 7). Viable osteocytes were present near the anchor surface (Fig. 8). No difference in the amount of bone anchor apposition was noted among specimens. Inflammatory cell infiltrate and foreign giant cell reactions were evident in three specimens containing titanium anchor and were not demonstrated in specimens with polylactic anchors. Also in group B no particulation or fracture with any of the anchors was revealed. In both groups there were regions of direct bone contact on all surfaces of the anchors.

The mechanical assessment of the anchor function were performed by observation of rabbit movement and response to external stimulation done by veterinary doctors, where no tendon repair failure were noticed among groups. From day 2 after surgery till the period of sacrifice the rabbits demonstrate a normal capability of movement and jump into their cages, free rest positions and good capability of alimentation. Statystical analysis (T-Test) was performed considering medium value, in both group, at day 40 and 120 , about inflammations cell count (number of inflammatory cell $/ \mathrm{mm}^{2}$ ), osteocyte count (number of histological evident osteocyte $/ \mathrm{mm}^{2}$ ), mineral apposi- 


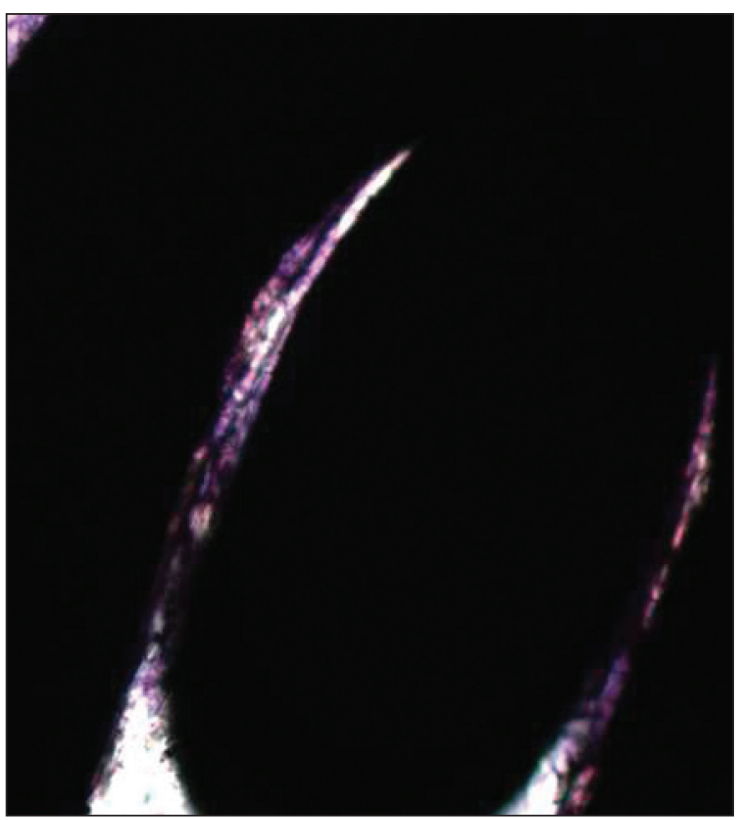

Figure 6. Group A. Particular of Titanium mini anchor implant at day 120. Implant was good embedded in bone.

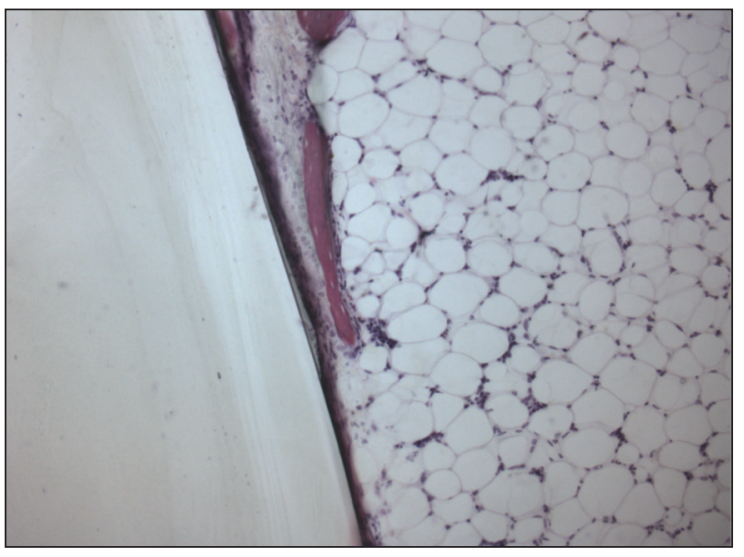

Figure 7. Group B. Polilactic mini anchor implant at day 120. The reabsorption is not present yet. No inflammation were observed (particular).

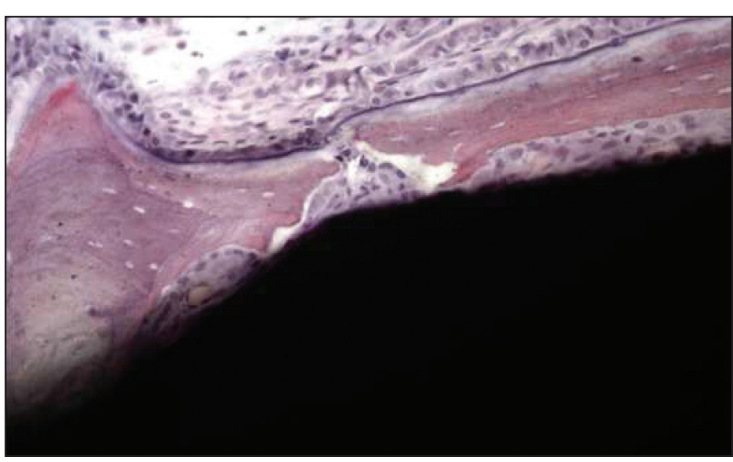

Figure 8. Group B. Polilactic mini anchor implant at day 120. Viable osteocytes were present near the anchor surface suggesting initials Osseointegration process. tion on anchor site (density range where evident at microscope). Significative difference was detected about inflammations $(p<0,05)$ demonstrating less foreign body response in reasorbeable groups. No significant difference $(p<0,05)$ were reported among groups in terms of osteocyte counts and mineral apposition (Tabs. 1-3).

\section{Discussion}

The tendon injuries represent an important challenge for hand surgery, particularly when it is not possible to perform a simple tendon suture but it is necessary to reinsert an avulse tendon. Therefore, many times, different techniques were created and evaluated for tendon fixation to the bone and with the help of technology by using an implant, especially miniaturized, like mini and micro suture anchor. The development of this technology conduced from a non reasorbeable implant (titanium) -that could have to be removed from the permanent implant, because of dislocation, infection or tissue reaction -to a reasorbeable implant (polylactic acid), that could avoid all these complications. In spite of the properties and the good results offered by the reasorbeable material ${ }^{18,19}$, there was not much scientific evidence about the results that could be obtained with its use in hand surgery in terms of Osseo integration, inflammatory response, fracture, in comparison with titanium anchors that are still widely more used than non reasorbeable ones, as we have done in our Hand Surgery Unit. This experimental study reproduced in an animal model a typical tendon injury and a repair using bone fixation by mini anchor. Histological evaluation showed that reasorbeable Mitek mini anchor Osseo integrates in different periods of healing, as well as non reasorbeable Mitek mini anchor, with less inflammatory response in a long period of time. We also observed that after 120 days non reasorbeable anchors were still present, suggesting that their reabsorption is still longer as declared by the Mitek Company, and it demonstrates that we could speak about a slow reabsorption anchor as reported in other studies.

Animal studies using LPLA implants have demonstrated degradation time to be at least 3 years, that was a not applicable period in our animal model for evaluation. Resorption of polymers generally occurs in two phases ${ }^{20}$. In the first phase, the polymer chains are broken down through hydrolysis. In this phase, the molecular weight drops first, followed by mechanical strength loss, and finally by a loss of mass $^{21}$. In the second phase, the implant loses its form and breaks physically into particles, which are attacked by macrophages. Depending on the size of the particulates, they are phagocytosed and the byproducts are excreted by the kidneys and lungs. The corresponding biological response to the degrading polymer is thought to happen as a result of either a build up of acidic degradation products or as a response to the particulates of the polymer22. The tim- 
Table 1. Inflammatory cells counts among two type of implants in rabbits sacrificed at day 40 and 120 (medium value).

\begin{tabular}{lll}
\hline $\begin{array}{l}\text { Rabbits on day } 40 \text { of sacrifice / } \\
\text { on day } 120\end{array}$ & $\begin{array}{l}\text { Tendon with reasorbeale material; } \\
\text { inflammatory cell/counts }- \text { mm }^{2}\end{array}$ & $\begin{array}{l}\text { Tendon with titanium implants; } \\
\text { inflammatory cell/counts }-\mathrm{mm}^{2}\end{array}$ \\
\hline Rabbit n.1 & $19 / 46$ & $17 / 34$ \\
Rabbit n.2 & $16 / 25$ & $19 / 27$ \\
Rabbit n.3 & $23 / 48$ & $25 / 45$ \\
Rabbit n.4 & $27 / 112$ & $24 / 110$ \\
Rabbit n.5 & $45 / 115$ & $39 / 117$ \\
Rabbit n.6 & $6 / 24$ & $8 / 23$ \\
Rabbit n.7 & $9 / 14$ & $7 / 13$ \\
Rabbit n.8 & $18 / 34$ & $17 / 38$ \\
Rabbit n.9 & $16 / 32$ & $15 / 37$ \\
Rabbit n.10 & $24 / 87$ & $25 / 88$ \\
\hline
\end{tabular}

Table 2. Viable osteocyte counts among two type of implants in rabbits sacrificed at day 40 and 120 (medium value).

\begin{tabular}{lll}
\hline $\begin{array}{l}\text { Rabbits on day } 40 \text { of sacrifice / } \\
\text { on day } 120\end{array}$ & $\begin{array}{l}\text { Tendon with reasorbeale material; } \\
\text { Viable osteocyte- } \mathbf{m m}^{2}\end{array}$ & $\begin{array}{l}\text { Tendon with titanium implants; } \\
\text { viable osteocyte }-\mathrm{mm}^{2}\end{array}$ \\
\hline Rabbit n.1 & $29 / 67$ & $19 / 68$ \\
Rabbit n.2 & $25 / 113$ & $23 / 118$ \\
Rabbit n.3 & $28 / 115$ & $29 / 117$ \\
Rabbit n.4 & $28 / 116$ & $26 / 119$ \\
Rabbit n.5 & $45 / 78$ & $47 / 81$ \\
Rabbit n.6 & $14 / 64$ & $16 / 61$ \\
Rabbit n.7 & $18 / 65$ & $20 / 70$ \\
Rabbit n.8 & $56 / 119$ & $57 / 121$ \\
Rabbit n.9 & $18 / 45$ & $19 / 49$ \\
Rabbit n.10 & $25 / 58$ & $25 / 56$ \\
\hline
\end{tabular}

Table 3. Mineral apposition ( $\mathrm{mm}$ ) among two type of implants in rabbits sacrificed at day 40 and 120 (medium value).

\begin{tabular}{lll}
\hline $\begin{array}{l}\text { Rabbits on day } 40 \text { of sacrifice / } \\
\text { on day } 120\end{array}$ & $\begin{array}{l}\text { Tendon with reasorbeale material; } \\
\text { mm mineral apposition }\end{array}$ & $\begin{array}{l}\text { Tendon with titanium implants; } \\
\text { mm mineral apposition }\end{array}$ \\
\hline Rabbit $\mathrm{n} .1$ & $0.04 / 0.07$ & $0.05 / 0.06$ \\
Rabbit n.2 & $0.03 / 0.08$ & $0.04 / 0.09$ \\
Rabbit n.3 & $0.01 / 0.03$ & $0.03 / 0.04$ \\
Rabbit n.4 & $0.04 / 0.06$ & $0.03 / 0.06$ \\
Rabbit n.5 & $0.06 / 0.08$ & $0.06 / 0.07$ \\
Rabbit n.6 & $0.04 / 0.05$ & $0.05 / 0.07$ \\
Rabbit n.7 & $0.05 / 0.09$ & $0.05 / 0.08$ \\
Rabbit n.8 & $0.04 / 0.06$ & $0.03 / 0.06$ \\
Rabbit n.9 & $0.05 / 0.09$ & $0.04 / 0.07$ \\
Rabbit n.10 & $0.03 / 0.09$ & $0.04 / 0.08$ \\
\hline
\end{tabular}

ing of the foreign-body response is thought to be related to the final stage of polymer degradation, so it explain as in our histological findings it is not represented. Anyway, according to our clinical opinion, a long time of reabsorption is more useful to permit a better Osseo integration and a good stress resistance, offering the opportunity for a better and more stable tendon healing, even also with low inflammatory response that could optimize tendon repair. Experimental studies cannot provide conclusions that are directly applicable to making decisions about the choice of material for the application to humans injuries. Nevertheless, tendon reinsertion by suture anchor is simpler and was demonstrated to be efficient in tendon avulsion and the use of a reasorbeable material could be considered similar to a non reasorbeable one with, moreover, a less inflammatory response in the period we have considered.
It could be interesting to evaluate, in a larger period of time, the reabsorption modality and how the new bone, that we observe in its first time of growth, could substitute the polylactic anchor. At this time of our study we could suggest that reasorbeable Mitek mini anchors offered the same security of non reasorbeable ones in terms of Osseo integration, stable fixation and, at the same time, avoided the possible complications linked to the use of a permanent implant. Besides we encouraging further experimental or clinical trial that could support our in vivo and histological observation.

\section{References}

1. Goble EM, Somers WK, Clark R, Olsen RE. The development of suture anchors for use in soft tissue fixation to bone. Am J Sports Med 1994 Mar-Apr; 22(2):236-239. 
2. Bhagia SM, Ali MS. Bankart operation for recurrent anterior dislocation of the shoulder using suture anchor. Orthopedics 2000; 23(6):589-591.

3. Lynch SA, Beard DM, Renström PA. Repair of distal biceps tendon rupture with suture anchors. Knee Surg Sports Traumatol Arthrosc 1999; 7(2):125- 131.

4. McDermott TP, Levin LS. Suture anchor repair of chronic radial ligament injuries of the metacarpophalangeal joint of the thumb. J Hand Surg 1998; 23B(2):271- 274.

5. Dzwierzynski WW, Sanger JR, Larson DL. Use of Mitek suture anchors in head and neck reconstruction. Ann Plast Surg $1997 ; 38(5): 449-454$.

6. Francis KR, Hoffman LA, Cornell C, Cortese A. The use of Mitek anchors to secure mesh in abdominal wall reconstruction. Plast Reconstr Surg 1994; 93(2):419-421.

7. Südkamp NP, Kääb MJ. Biodegradable implants in soft tissue refixation: experimental evaluation, clinical experience, and future needs. Injury 2002; 33(Suppl 2).

8. Ambrose CG, Clanton TO. Bioabsorbable implants: review of clinical experience in orthopedic surgery. Ann Biomed Eng 2004; 32:171-177.

9. Kulkarni RK, Pani KC, Neuman C, Leonard F. Polyactic acid for surgical implants. Arch Surg 1966; 93:839-843.

10. Baeber FA, Herbert MA, Richards DP. Sutures And suture anchors : update 2003. Arthroscopy 2003; 9:109-113.

11. Bunnel S. Gig pull-out suture for tendons. J Bone Joint Surg 1954; 36A:850-851.

12. Guinard D, Montanier F, Thomas D, Corcella D, Moutet F. The Mantero flexor tendon repair in zone 1. J Hand Surg 1999; 24B:755-757.

13. Giannikas D, Athanaselis E, Matzaroglou C, Saridis A, Tyl- lianakis M. An unusual complication of Mitek suture anchor use in primary treatment of flexor digitorum profundus tendon laceration: a case report. Cases J 2009; 2:9319.

14. Claes L, Ignatius A. Development of new, biodegradable implants. Chirurg 2002; 73:990-996.

15. Shaieb MD, Singer DI, Grimes J, Namiki H. Evaluation of tendon -to-bone re attachment: a rabbit model. Am J Orthop 2000; 29:537-542.

16. Matos MA, Tannuri U, Guarniero R. Effect of ifosfamide on bone healing. J Muscoloskeletal Neuronal Interact 2006; 6:247-250.

17. Donath K, Breuner G. A method for the study of undecalcified bones and teeth with attached soft tissues. The SägeSchliff (sawing and grinding) technique J Oral Pathol 1982 11:318-326.

18. Madhavan Nampoothiri K, Nair NR, John RP. An overview of the recent developments in polylactide (PLA) research. Bioresour Technol 2010; 101:8493-8501.

19. Kontakis GM, Pagkalos JE, Tosounidis TI, Melissas J, Katonis P. Bioabsorbable materials in orthopaedics. Acta Orthop Belg 2007; 73(2):159-169.

20. Kohn J, Langer R. Bioresorbable and bioerodable materials. In: Biomaterials Science: An Introduction to Materials in Medicine, edited by B. D. Ratner, A. S. Hoffman, F. J. Schoen and J. E. Lemons. San Diego, CA: Elsevier 1996; 64-72.

21. Pietrzak WS, Sarver D, Verstynen M. Bioresorbable implantspractical considerations. Bone 1996; 109S-119.

22. Bergsma JC, De Bruijn WC, Rozema FR, Bos RRM, Boering $\mathrm{G}$. Late degradation tissue response to poly (I-lactide) bone plates and screws. Biomaterials 1995; 16:25-31. 